\title{
Responsabilidade social em oftalmologia: interdisciplinaridade e inclusão na visão subnormal
}

\section{Social responsibility in ophthalmology: interdisciplinarity and inclusion in low vision}

\author{
José Américo Bonatti', Marcos Wilson Sampaio', Fernanda \\ Alves da Silva Bonatti ${ }^{2}$, Maria Cecília Loschiavo dos Santos ${ }^{3}$, \\ Newton Kara-José ${ }^{4}$
}

\begin{abstract}
Bonatti JA, Sampaio MW, Bonatti FAZ, Santos MCL, Kara-José N. Responsabilidade social em oftalmologia: interdisciplinaridade e inclusão na visão subnormal. Rev Med (São Paulo). 2007 out.-dez.;86(4):195-200.
\end{abstract}

RESUMO: Há ainda hoje uma importante exclusão das pessoas deficientes visuais, principalmente das de baixa visão (em especial daqueles com visão corrigida entre 0,3 e 0,05 no melhor olho). É importante que a família, a escola, a sociedade e o/a paciente participem deste processo de inclusão social para proporcionar uma vida próxima da normal para essas pessoas com baixa visão. Deve haver uma interação entre os educadores e o paciente, a família (para apoio psicológico e continuação da reabilitação em casa) e o oftalmologista para otimizar o uso dos equipamentos visuais para as atividades cotidianas do paciente na escola, trabalho, lazer ou em outras situações. É também importante uma interação entre a oftalmologia e profissionais de design para fornecer aparelhos de auxílio o mais ergonômicos possíveis para permitir ao paciente com baixa visão uma adaptação adequada à vida cotidiana. Na Universidade de São Paulo, o Departamento de Oftalmologia da Faculdade de Medicina iniciou uma colaboração com membros do Departamento de Design da Faculdade de Arquitetura e desenvolveram um equipamento óptico que torna a leitura mais fácil e confortável para os pacientes com baixa visão acima citados. O método de trabalho foi baseado na integração de conhecimento, pensando através das disciplinas, assim como na observação participativa das necessidades dos pacientes na sua vida diária. Este grupo propôs um equipamento ergonômico para permitir um melhor desempenho visual dos pacientes, baseado nas suas necessidades, visando sua inclusão numa vida educacional, familiar e social a mais normal possível. Este é apenas o começo de uma interação que vai continuar, com o objetivo de desenvolver outros aparelhos ópticos e eletrônicos e mesmo mobília para permitir a esses deficientes visuais um melhor desempenho no seu dia a dia.

DESCRITORES: Responsabilidade social. Baixa visão/reabilitação. Transtornos da visão/ psicologia. Portadores de deficiência visual/psicologia. Oftalmologia/instrumentação.

1. Doutor em Medicina da Clínica Oftalmológica do Hospital das Clínicas da Faculdade de Medicina da Universidade de São Paulo (HCFMUSP).

2. Médica, Arquiteta e Doutoranda em Design e Arquitetura da Faculdade de Arquitetura e Urbanismo da USP (FAUUSP).

3. Professora Associada, Pesquisadora do CNPQ, da Faculdade de Arquitetura e Urbanismo da USP (FAUUSP)

4. Professor Titular. Doutor em Medicina da Clínica Oftalmológica do Hospital das Clínicas da Faculdade de Medicina da Universidade de São Paulo (HCFMUSP).

Endereço para correspondencia: Rua Teodoro Sampaio, 744, Cj.106. CEP: 05406-000. E-mail: bonatti@uol.com.br 
A pós tantos avanços em relação à inclusão social dos deficientes, os deficientes visuais ainda permanecem excluídos parcial ou totalmente de uma vida social digna e satisfatória, tanto que é necessário legislarse para favorecer sua inclusão social, delegando à família, à escola e à sociedade o compromisso de proporcionar uma escola para todos, além de estimular a acessibilidade e inclusão destes indivíduos nos locais de suas atividades diárias, no estudo, num trabalho compatível com suas potencialidades e desejo, no lazer e em outras atividades sociais.

Um deficiente visual que se defronte com barreiras no seu entorno e em suas próprias limitações tende a se tornar frustrado, deprimido e posteriormente isolado após tentativas infrutíferas de conviver em sociedade ${ }^{1}$. Daí a importância da família, da escola, da sociedade e de entidades promotoras de inclusão social, públicas e privadas, no estabelecimento de um ambiente favorável para que este indivíduo possa encontrar um caminho digno, acolhedor de suas limitações e promotor de condições para que este deficiente se desenvolva e possa, não só ter uma formação escolar, uma profissão, um emprego, uma vida social, familiar e afetiva humanisticamente adequadas, que Ihe tragam auto-estima e qualidade de vida, mas que também possa ter suas ações se refletindo positivamente no mundo ao seu redor, trazendo-lhe uma sensação de participação e integração ao mundo à sua volta. Evidentemente, até para os que não são deficientes, este ideal a ser atingido é difícil. Contudo se não se planeja atingir este objetivo, atinge-se muito menos e quando se fala de deficientes visuais, em condições de desigualdade óbvias num mundo competitivo como é o nosso, deve-se-lhes proporcionar as melhores condições possíveis para integração social, para que o degrau que os separa dos não deficientes tenda a diminuir. Parafraseando Don Guanella, um religioso italiano que implantou entidades de assistência e educação para deficientes carentes espalhadas por todo o mundo, "deve-se dar o melhor para os que menos têm".

Dentro desta lógica, do ponto de vista operacional, o que se deve proporcionar de ensinamento e convívio a essas pessoas deve levar em conta o grau de deficiência visual que apresentam, daí a importância inicialmente do conhecimento do quadro clínico, através da avaliação oftalmológica, que irá determinar quão profunda é a deficiência visual. Essa avaliação irá orientar as ações de habilitação e reabilitação levadas a cabo pelo deficiente, pela família e pela escola, isoladas e conjuntamente. Outro aspecto a ser simultaneamente avaliado é o do grau de percepção que esses indivíduos possuem de se sentirem capazes para realizar atividades variadas do cotidiano, ou seja, a percepção de sua auto-eficácia. A percepção de alta ou baixa eficácia em determinada atividade terá reflexo direto na autoestima do deficiente visual e conseqüentemente na sua qualidade de vida. Já existem estudos importantes da percepção, pelo deficiente visual, nas atividades da vida diária, da facilidade ou dificuldade para realizar essas atividades, a percepção das causas dessas dificuldades e o levantamento da realização ou não destas atividades, permitindo formar um quadro amplo de conhecimento sobre o deficiente visual, envolvendo desde o auto julgamento em relação à própria capacidade e desembocando na realização da tarefa mesma, permitindo a planejadores de habilitação, reabilitação e de inclusão de deficientes visuais propor ações mais realistas.

A definição de deficiência, que abrange desvio significativo ou perda de função nas estruturas do corpo, enquanto funcionalidade abrange todas as funções do corpo, a atividade e a participação. Clinicamente, baixa visão ou visão subnormal é a visão menor que 20/60 e cegueira é a visão menor que $3 / 60$ ou 0,05 . Há uma curiosa discrepância entre o conceito educacional e o conceito da Organização Mundial da Saúde (OMS) de cegueira, pois segundo os educadores cegueira é entre percepção de luz sem projeção luminosa (SPL), o que pode dificultar muito a locomoção, enquanto para a OMS é visão menor que 0,05 , que ocorrendo em indivíduo com retina periférica boa, pode ter projeção de luz e locomoção razoável. Além destes há o conceito legal de cegueira, que é visão menor que 0,1 . Seria interessante, portanto, a uniformização destes conceitos.

A perda de visão é um problema do indivíduo e da coletividade, que devem interagir, juntando o indivíduo deficiente, a família e a escola para melhorar seu desempenho nas tarefas cotidianas, escolares e intelectuais, aumentando sua sensação de independência, de capacidade de realização e de conhecimento, com reflexos positivos na sua auto estima, melhorando sua qualidade de vida e inserindo-o socialmente. Por outro lado a dificuldade de desempenhar as tarefas pessoais e a dependência são devastadoras para o indivíduo do ponto de vista psíquico, social e financeiro.

As necessidades básicas humanas de alimentação, sono, repouso e posteriormente de segurança social, afetiva, de status, de estima e de auto-realização constituem o ponto de partida para a ação e também para satisfação de outras necessidades e uma deficiência, notadamente a visual, pode dificultar determinada ação, pois a visão é responsável por $85 \%$ do contato do indivíduo com o mundo. 
Insere-se muito adequadamente neste contexto, portanto, o conceito de auto-eficácia ${ }^{1}$, como crença na capacidade de exercer controle sobre o próprio funcionamento e o ambiente, enfrentando o desconhecido, diminuindo a vulnerabilidade, o stress e a depressão, adotando uma postura otimista em relação à vida, elevando a autoestima e melhorando em última análise a qualidade de vida. Tem implicações no autoconceito e na autoestima, sendo o juízo das próprias habilidades. Deve-se reiterar estas palavras por ser para os médicos conceitos relativamente novos e certamente pouco utilizados e por terem importância fundamental na integração digna do deficiente no mundo à sua volta. Está ligada à repetição bem sucedida, sendo motivadora de novas ações. Quem tem percepção de auto-eficácia positiva adota comportamento a favor da saúde, modificando comportamentos prejudiciais. Lembrando a definição de saúde da OMS, que é o bem estar físico, mental e social, a percepção de autoeficácia tem implicações psíquicas, com repercussões físicas e sociais, deste modo sendo um grande balizador nesta área. A capacidade de realizar as atividades da vida diária (AVD) é fundamental para se avaliar o grau de independência do deficiente visual, com repercussões diretas sobre sua qualidade de vida, pois contribui para aquisição de conhecimento, independência e autonomia.

Respeitando-se os limites do deficiente bem treinado e habilitado, dever-se-ia inverter esta lógica perversa e moralmente inaceitável de exclusão dos que têm menos e de inclusão dos que têm melhores condições. O processo de reabilitação em escolas adequadamente preparadas aumenta a conscientização de seu problema, facilitando o desenvolvimento em direção a uma melhor qualidade de vida. Viver somente com a família parece ser um limitador da independência, mesmo que, aparentemente, diminua a sensação de solidão. Portanto um caminho é integrar a família à reabilitação, conscientizando-a da necessidade de promover o autodesenvolvimento dos seus filhos deficientes.

Apesar de a maioria ter auto-eficácia para freqüentar escola e se suceder bem, parte expressiva dos deficientes visuais tem dificuldade com computador e Internet, talvez por barreiras sociais, culturais e econômicas, que deveriam merecer a atenção dos educadores. Uma sugestão seria as escolas terem, além de uma casa simulada ou casa piloto para treinamento precoce a partir da educação infantil para o aprendizado de AVD, uma área para aprendizado com computadores.

É fundamental a atuação conjunta desde o início do deficiente, da escola e da família, para que estes três atores otimizem a reabilitação, pois esta influencia diretamente o nível de escolaridade.

Enfim, pode-se dizer que o portador de visão sub-normal fica num limbo (ou seria purgatório?) entre os videntes e os cegos, necessitando de maior atenção em termos de auxílios ópticos e de apoio psicológico e pedagógico.

A independência leva à autoconfiança e a novas iniciativas, daí a necessidade de inclusão e reabilitação, já existindo estudos que fornecem importantes subsídios para o estabelecimento de políticas de saúde para o deficiente visual.

Um dos setores em que se deve atuar para a inclusão do portador de visão subnormal é o de design de equipamento adequado para leitura.

A contribuição que a Clínica Oftalmológica do Hospital das Clínicas da Faculdade de Medicina da USP em associação com a Faculdade de Arquitetura e Urbanismo da USP pode oferecer já ficou evidenciada. A Dra . Fernanda Alves da Silva Bonatti, uma das autoras deste trabalho, médica formada pela USP, levantou, catalogou e sistematizou a problemática vivida pelo paciente com visão subnormal no setor de Visão Subnormal do HCFMUSP, chefiado pelo Prof. Dr. Marcos Wilson Sampaio, que diagnostica e trata doentes com visão subnormal. Tal observação teve como objetivo o desenvolvimento de um equipamento de auxílio à visão subnormal para leitura, tema de seu trabalho final de Graduação pela Faculdade de Arquitetura e Urbanismo da USP², cuja banca teve a participação do Prof. Dr. Marcos Wilson Sampaio, da FMUSP e da Profa. Titular da FAUUSP Élide Monzéglio. Apresentado em fevereiro de 2005, sua orientação foi realizada pela também autora deste trabalho, a Prof ${ }^{a}$. Dr ${ }^{\text {a }}$. Associada da FAAUSP Maria Cecília Loschiavo dos Santos, pesquisadora de projeção nacional e internacional na área do Design e Responsabilidade Social. A proposição da Dra . Fernanda foi baseada não só nas necessidades ópticas, mas também nas necessidades de adaptar a máquina ao homem (ergonomia), resultando num equipamento com características funcionais e estéticas adequado à realidade de nosso meio para permitir a leitura de textos corriqueiros, como jornais, livros, cadernos e revistas com conforto sem perder a linha de leitura. Este desenvolvimento teve uma característica de interface de duas áreas complementares e de interdisciplinaridade, por ter aliado os conhecimentos da Faculdade de Arquitetura e ter envolvido situações médicas. Devese, portanto, refletir que este tipo de iniciativa pode e deve continuar a ser praticado neste tão carente setor no nosso país, pois os problemas do deficiente visual muitas vezes necessitam desta abordagem 
multidisciplinar para terem suas soluções otimizadas, bem como para fomentar o design de produtos que não estigmatizem seus usuários. É importante a continuidade deste tipo de iniciativa de interação entre estas duas áreas, visto a Clínica Oftalmológica do HCFMUSP já ter tradição no desenvolvimento de equipamentos, mas de maneira isolada, sem a contribuição de outras áreas. Podemos citar na área de Bioengenharia desenvolvimentos em que Médicos desta Clínica estiveram envolvidos, apenas à guisa de exemplo parcial, pois certamente não estaremos nos lembrando de todos:

> Estesiômetro (Prof. Dr. Newton Kara-José);

> Vitreófago (Prof. Dr. Hisashi Suzuki);

> Crioextrator para cirurgia de catarata (Prof. Dr. Hisashi Suzuki);

> Diatermocoagulador bipolar ${ }^{11}$ (Prof. Dr. Hisashi Suzuki);

- Gancho de íris para dilatação mecânica da pupila $^{12}$ ( Prof. Dr. Hisashi Suzuki);

> Implante para glaucoma (Prof. Dr. Remo Susanna Jr.);

$>$ Aperfeiçoamento de crioextator (Dr. José A.Bonatti e Dr. José C. E. Carani);

> Oftalmoscópio binocular indireto nacional ${ }^{4}(\mathrm{Dr}$. José A. Bonatti);

> Videoceratoscópio para análise topográfica da córnea ${ }^{7}$ (Dr. José A. Bonatti);

> Equipamento de geração e registro de pressão intra-ocular para uso experimental em testes de perfurações corneanas tamponadas com adesivo ${ }^{3}$ (Dr. José Américo Bonatti, Prof. Dr. Hisashi Suzuki, Prof. Dr. Newton Kara-José);

> Telelupa para visão subnormal10 (Dr. Marcos Wilson Sampaio, Dra. Maria Aparecida Onuki Haddad).

As iniciativas acima elencadas certamente ocorreram por obra e engenhosidade individual e depois foram desenvolvidas em equipe, mas certamente não seguiram uma diretriz estratégica maior determinada pelas áreas médica e de design, como meta a ser alcançada dentro da área de bioengenharia.

A Clínica Oftalmológica do HCFMUSP possui a melhor e mais abrangente medicina assistencial possível dentro do setor público brasileiro, tanto na área clínica como cirúrgica, possui uma PósGraduação nível Doutorado modelo, dentro da qual são desenvolvidas excelentes teses, tanto clínicas como experimentais, forma Oftalmologistas do mais alto gabarito em sua Residência Médica, promove o ensino de Oftalmologia no curso de Graduação da FMUSP além de Congressos como parte de sua atuação na extensão de ensino à comunidade Oftalmológica.

Como se pode ver, existe nesta Clínica um grande trabalho já desenvolvido e em desenvolvimento, já que se trata de uma clínica universitária cheia de dinamismo. Uma diretriz estratégica focada no desenvolvimento de produtos decorrentes da bioengenharia, com design biomédico focado em oftalmologia para o portador de visão subnormal certamente traria vantagens à Clínica, pois racionalizaria os recursos e os esforços nesta área específica que é complementar à atividade diária da Oftalmologia Clínica. Esta área teria condições de propor soluções para problemas clínicos vividos em nosso meio, que hoje ou não são solucionados ou o são com tecnologia cara e importada. Isto decorre de a Oftalmologia ser uma área de rápido progresso, porém a alto custo, visto sermos dependentes da cara tecnologia estrangeira na maioria das vezes.

A criação de um grupo de design oftalmológico nesta Clínica para desenvolver projetos de bioengenharia viria atender às demandas assistenciais ao paciente da área Oftalmológica, notadamente na área de visão subnormal. Certamente é um trabalho complexo, que demandará recursos, pessoal da área Oftalmológica e de Design, designers e pessoal da área médica formados e em fase de aprendizado (alunos de iniciação científica), pessoal especializado de diversas áreas, além de técnicos especializados.

Tal grupo, além de ter Oftalmologistas e Arquitetos, contaria também com pessoal especializado de áreas complementares, como Física, Química, Engenharia, da própria Universidade de São Paulo e importantes na resolução de problemas de interface de áreas apresentados por estes tipos de projetos. A interdisciplinaridade com o objetivo de realizar projetos na área da saúde vêm de encontro a discussões e preocupações internacionais da atualidade. Rachel Cooper, professora da Universidade de Salford, na Inglaterra, e presidente da Academia Européia de Design atenta para as responsabilidades sociais do designer, que inclui tanto a preocupação com a sustentabilidade quanto a acessibilidade e a inclusão. Diz ela que designers são essenciais para trazer a responsabilidade social para a vida.

Cooper ${ }^{5}$, em artigo publicado no ano de 2005, identifica um movimento que define como "Corporate Social Responsibility" (CSR) que está sendo promovido por organizações governamentais e por empresas privadas de diversos países da Europa e da América do Norte no sentido de colocar em prática o crescimento sustentável, a inclusão social e a responsabilidade perante as comunidades particularmente no que diz respeito à saúde e à 
educação. O tema Design Social começou a ganhar espaço a partir da década de 1960, quando críticos de design passaram a abordar assuntos como o consumismo, o design "verde", o ecodesign, a sustentabilidade e o design direcionado às mulheres. A acessibilidade e a inclusão passaram a fazer parte do interesse desse tipo de abordagem do design, tornando-se campos de trabalho e de negócios. A responsabilidade social deriva, em parte, dos valores éticos individuais dos designers, mas é também uma resposta às necessidades da população $0^{6,8,9,13}$.

Dentro da Clínica Oftalmológica e da Faculdade de Arquitetura, poder-se-ia contar com a participação de Professores e Doutores para orientação dos trabalhos, pós-graduandos interessados em desenvolver projetos nesta área, residentes que se interessem pelo tema e alunos do curso de Graduação com interesse em iniciação científica.

Com a estruturação deste grupo, certamente ficaria mais fácil o contato e o trabalho conjunto da
Clínica Oftalmológica com as áreas complementares acima citadas dentro da Universidade, que certamente apresentariam algum nível de resposta e solução para os problemas propostos na Clínica Oftalmológica nesta área, trabalho que seria muito mais difícil se realizado isoladamente. $\mathrm{O}$ trabalho conjunto com estas áreas certamente facilitaria a proposição de projetos temáticos de peso aos órgãos financiadores de pesquisa e aumentaria a chance de aprovação, pois estariam bem consolidados e cientificamente viáveis.

A criação deste grupo resultaria num trabalho interdisciplinar, focado em design de equipamento oftálmico para o portador de visão subnormal, abrindo a possibilidade de um vasto leque de pesquisas, contribuindo para aumentar o número de publicações científicas e para o desenvolvimento tecnológico no nosso país, tornando a Clínica Oftalmológica do HCFMUSP e as pesquisas sobre design social da FAUUSP ainda mais visíveis dentro do campo científico.

Bonatti JA, Sampaio MW, Bonatti FAZ, Santos MCL, Kara-José N. Social responsibility in ophthalmology: interdisciplinarity and inclusion in low vision. Rev Med (São Paulo). 2007 out.-dez.;86(4):195-200.

\begin{abstract}
There is still today an important exclusion of the visually impaired people, mainly the low vision ones (particularly people with corrected vision between 0,3 and 0,05 in the better eye). It is important that the family, the school, the society and the patient him/herself participate in this process of social inclusion to provide a near normal life for these low vision people. There must be an interaction between the educators and the patient, the family (for psychological support and continuing the home rehabilitation) and the ophthalmologist for optimizing the use of visual devices for the patient's daily activities at school, work, leisure or whatsoever. It is also important an interaction between the ophthalmology and design professionals for providing the most ergonomic as possible devices to permit the low vision patient an adequate adaptation to the everyday life. In the University of São Paulo/Brazil the Ophthalmology Department of the Medical School initiated a collaboration with members of the Design Department of the Architecture School and developed an optical device that can make reading easier and more comfortable for low vision patients. The method of work was based in the integration of knowledge, in thinking across disciplines as well as in the participative observation of the patients' needs in their life. This group purposed an ergonomic equipment to permit a better visual performance of the patients, based on their needs, aiming their inclusion in an educational, familiar and social life as normal as possible. This is only the beginning of an interaction that will continue, with the goal of developing other optic and electronic devices and even furniture devices as to permit these visually impaired people a more adequate performance in their everyday life.
\end{abstract}

KEY WORDS: Social responsability. Vision, low/rehabilitation, Vision disorders/psychology. Visually impaired persons/psychology.

\title{
REFERÊNCIAS
}

1. Arruda SMCP. Percepções da auto-eficácia nas atividades de vida diária e qualidade de vida de estudantes com baixa visão ou cegueira. [tese]. Campinas: Faculdade de Ciências Médicas da Universidade Estadual de Campinas; 2005.
2. Bonatti FAS. Desenvolvimento de equipamento de auxílio à visão subnormal. Arq Bras Oftalmol. 2006;69(2):221-6.

3. Bonatti JA, Suzuki H, Kara-José N, Matheus LCA. Desenvolvimento de equipamento de geração e registro 
Bonatti JA et al. Responsabilidade social em oftalmologia: interdisciplinaridade e inclusão na visão subnormal.

de pressão intraocular suportada por perfuração corneana colada com fibrina. Arq Bras Oftalmol. 1997;60(5):514-5.

4. Cavalcante I. Ataque à miopia com raio laser. J USP. 1991;5(171):12.

5. Cooper R. Ethics and altruism: what constitutes socially responsible design? In: Design Management Review Summer. 2005; 16(3):10-8.

6. Ethos. Instituto de Empresas e Responsabilidade Social. Responsabilidade social das empresas: a contribuição das universidades. São Paulo: Editora Petrópolis; 2003. v.2.

7. Oliveira AC, Yasuoka FMM, Tonissi-JR SA, Cansian AM, Ramos JEB, Romão AC, et al. In: Encontro Nacional de Física da Matéria Condensada, 15. Programa e resumos. São Paulo: Sociedade Brasileira de Física;
1992. Caxambu, Brasil; 1992. p. 157.

8. Papanek V. Diseñar para el mundo real-ecologia humana y cambio social. Madrid: Blume Ediciones; 1977.

9. Papanek V. Arquitectura e design. Lisboa: Edições 70; 1995.

10. Sampaio MW et al. Auxílios para baixa visão. São Paulo: Laramara; 2001.

11. Suzuki H, Suzuki R, Suzuki CR. Diatermocoagulador bipolar: modo de construção. Arq Bras Oftalmol. 1995;58(3):175-6.

12. Suzuki H, Suzuki R, Suzuki CR. Gancho de íris para dilatação mecânica da pupila: modo de construção. Arq Bras Oftalmol. 1995;58(5):367-8.

13. Whiteley N. Design for society. London: Reaktion Books; 1993. 